\title{
The Effects of Opioids During Pregnancy: A Literature Review
}

\author{
Saman Asdjodi ${ }^{1}$ a, Rodrigo B Rubarth ${ }^{1}$ b, Joshua Hardy $^{1}$, Harry Lee ${ }^{1}$ d \\ ${ }^{1}$ Georgetown University School of Medicine \\ Keywords: maternal opioid use, developmental defects, embryogenesis \\ https://doi.org/10.52504/001c.16759
}

\section{Georgetown Medical Review}

Vol. 4, Issue 1, 2020

\begin{abstract}
The alarming increase in opioid use in the United States, particularly during pregnancy, over the past few decades underlines the need to thoroughly investigate the consequences of opioid use within the context of reproduction and development. Opioid exposure has been linked to a number of effects on the various physiologic processes involved in embryonic development. Opioids have been shown to hinder the preimplantation embryo from progressing into the blastocyst stage and implanting into the uterus. Maternal opioid use has also been shown to be neurotoxic to the embryo. Exogenous opioids negatively affect the somatosensory cortex, hippocampus, and cholinergic system in the developing embryo, leading to consequences ranging from poor memory function to learning disabilities. Additionally, opioids have the potential to negatively affect the embryonic heart. Opioid use has been shown to slow down the growth of cardiac tissue, decrease fetal heart rate, and increase the incidence of congenital heart defects. Through review of existing studies, we conclude that opioid use during pregnancy has a significant risk of being detrimental to the embryo. Based on the available scientific literature, we recommend reevaluating current guidelines on opioid use during pregnancy to ensure that opioid exposure to the embryo is limited as much as possible.
\end{abstract}

\section{Introduction}

Opioid use in the United States has dramatically increased over the last 2 decades and opioid prescription rates in the United States are almost 4 times higher than rates in Europe. ${ }^{1}$ A previous study found that across different states, $9.5 \%$ to $43.6 \%$ of Medicaid-enrolled pregnant women received at least 1 opioid prescription during their pregnancy. ${ }^{2}$ Although opioids have not been directly associated with teratogenicity at prescribed doses, their use during pregnancy remains a controversial medical recommendation given the risk for misuse and abuse. Studies have shown that $8 \%$ to $12 \%$ of opioid prescriptions result in opioid misuse and that $4 \%$ to $6 \%$ of people who misuse opioid prescriptions transition to heroin use. ${ }^{3}$

The risk for post prescription opioid abuse is especially concerning if the person receiving the prescription is pregnant, given that prenatal opioid exposure has been shown to have negative effects on the offspring's physical and mental performance well into childhood and adulthood. In neonates, such

\footnotetext{
a Authors contributed equally.

b Authors contributed equally.

c Authors contributed equally.

d Authors contributed equally.
} 
effects include reduced ability to concentrate, increased incidence of apathy, reduced physical activity, and decrease visual acuity. ${ }^{4}$ Newborns born to heroin users are also more likely to be premature, which increases their risk for lifethreatening complications in the neonatal period. ${ }^{4}$ Children born to mothers addicted to opioids are also more likely to show poor socialization, learning deficiencies, and reduced responsiveness as they grow older. ${ }^{5}$ Therefore, the increasing rates of opioid prescription and abuse in the United States, both of which affect the pregnant women population, pose serious public health concerns.

The scientific community plays a vital role in helping to alleviate the public health burden of increasing numbers of women using opioids during pregnancy. Key areas of focus include advancing research on interventions for overdose reversal, safe treatments for opioid addiction, and alternative nonaddictive treatments for pain. Another logical strategy is to better understand how opioids-both endogenous and exogenous-affect embryonic development.

The purpose of this article is to review scientific literature on how opioids influence biochemical pathways, and how exogenous opioid-induced morphological and physiological changes during embryogenesis may explain incidences of poor neonatal health outcomes and the impaired development of children born to opioid addicted mothers. The strength of association between maternal opioid use and neonatal health outcomes is impacted by a few confounding variables, such as the mother's nutrition and socioeconomic status. Nonetheless, there is enough evidence suggesting that opioid prescription during pregnancy significantly contributes to the growing public health concern regarding this drug class. To this point, our position is that guidelines for opioid prescriptions must be reassessed to reduce the pharmacological use of opioids during pregnancy due to the danger they pose to the developing embryo.

\section{Opioid Effects by Developmental Regions}

\section{Preimplantation}

It has been well established that opioid receptors can be found on sperm, follicular cells, oocyte cells, preimplantation embryos (zygote to blastocyst), and the mother's uterus. These receptors can be found on the membranes or in the cytoplasm of cells. 6,7 The 3 main opioid receptor subtypes in the opioid system, all of which are $G$ protein coupled $-\delta$ delta (Oprd/DOR), $x$ kappa $(\mathrm{OprK} / \mathrm{KOR})$, and $\mu \mathrm{mu}(\mathrm{OprM} / \mathrm{MOR})$. These receptors are found on the preimplantation embryo and preimplantation uterus. ${ }^{8}$ Further, different endogenous opioid peptides are derived from various precursors. Endorphins are made from proopiomelanocortin, enkephalins from preproenkepahlin, and dynorphins originate from preprodynorphin. ${ }^{6,9}$ In this process, synthetic prohormone convertase $1 / 3$ and prohormone convertase 2 serve a crucial role in regulating the production of opioids during the development of the 
preimplantation embryo. ${ }^{6,9}$ Understanding the activity of endogenous opioid ligands and their precursors provides insight into the effects of exogenous opioids on embryo preimplantation and uterine receptivity to blastocyst implantation.

When activated, opioid receptors inhibit depolarization via voltage-gated calcium ion $\left(\mathrm{Ca}^{2+}\right)$ channels. In the preimplantation embryo, disruptions in necessary $\mathrm{Ca}^{2+}$ mobilization through excessive stimulation of the $\mu$ receptor (MOR) leads to growth retardation. ${ }^{6}$ In one study, less than $50 \%$ of mouse embryos progressed to the blastocyst stage when treated with morphine, with most stopping their development at the 2-cell embryo stage. In comparison, $90.6 \%$ of mouse embryos successfully became a blastocyst in the control group treated with saline. ${ }^{6}$ The growth retardation of preimplantation embryos limits their successful implantation into the uterus.

Opioid signaling also influences the receptivity of the uterus to blastocyst implantation. More specifically, exogenous opioids attenuate luminal epithelial differentiation, reduce angiogenesis, and limit stromal cell proliferation. ${ }^{7}$ Mouse models show a high expression of opioid receptors in the uterine epithelium from day 1 of pregnancy and in the uterine stroma from day 4 of pregnancy. Morphine-treated pregnant mice were found to have a decrease in 3 important markers associated with uterine implantation receptivity: lactoferrin, mucin 1 , and leukemia inhibitory factor. ${ }^{10}$

Another study found that morphine-treated pregnant mice also showed decreased expression of progesterone-responsive genes found in the uterine stroma, such as Hand2, Hoxa-10, and Coup-TFII. ${ }^{7}$ The decreased expression of these genes indicates a disruption in progesterone's physiologic action of stimulating angiogenesis in the uterine endometrium. As a consequence, the proliferation of stromal cells necessary for successful blastocyst implantation was limited, as evidenced by reduction in measured platelet endothelial cell adhesion molecule1, which is a marker for blood vessel density in the uterine stromal bed. ${ }^{7}$ These findings suggest that exogenous opioid use increases rates of implantation failure.

The aforementioned observations of opioids affecting the preimplantation stages of pregnancy and embryonic development are further strengthened by findings that the coadministration of the opioid receptor antagonist naloxone can mitigate morphine's inhibitory effects on preimplantation embryonic growth and endometrial stromal cell proliferation. By inhibiting the effects of morphine on opioid receptors, especially $\mu(\mathrm{OprM}), \mathrm{Ca}^{2+}$ mobilization was able to increase to normal levels and physiologic preimplantation signaling was restored. ${ }^{6}$ In addition, inhibiting the effects of morphine on progesteroneresponsive genes allowed for the physiologic priming of the uterine lining for implantation by proper luminal epithelial differentiation, increased angiogenesis in the endometrium, and increased stromal cell proliferation. ${ }^{7}$ 
It has been shown in mouse models that long-term morphine treatment can derail the hypothalamic-pituitary-gonadal axis and, thus, negatively affect gonadal steroidogenesis leading to poor uterine development. ${ }^{11}$ More recent studies have shown that mice that undergo short-term morphine treatment have normal gonadal steroidogenesis but also have poor uterine development due to intrinsic uterine defects caused by excess stimulation of opioid receptors. ${ }^{7}$ This is another reason that guidelines for opioid prescriptions for women who are pregnant or planning to become pregnant should be reassessed, and more caution should be taken before these women are prescribed opioids-even for short-term use-until more research can be conducted.

\section{Central Nervous System}

It is understood that endogenous opiates play an important role in the normal development of several regions of the brain by influencing cell proliferation, neurite and dendritic outgrowth, and overall neuronal density. ${ }^{12}$ In general, opiates are thought to have an inhibitory effect on these measures of development. The effects of endogenous opioids on the development of the central nervous system are fairly diffuse. In fact, a study using mice models found that prenatal exposure to opioids resulted in offspring having reduced numbers of brain cells, as well as deficits in RNA and protein levels in brain tissue. ${ }^{5}$ Disruptions in physiologic opioid-receptor signaling by exogenous opioids are likely to cause a variety of biochemical alterations that may be involved in the aforementioned neurobehavioral sequelae found in higher incidence among newborns and children prenatally exposed.

More specifically, one study measured the influence of opioid receptor activity on cortical cell packing density, thickness, and cell number in specific layers of the primary somatosensory cortex during development, where opioid receptor signaling has been shown to play a particularly significant role in cortical neuron development. ${ }^{12}$ This study investigated the effects of endogenous and exogenous opiates on brain development through continuous administration of naltrexone or morphine during the period of cortical neuron proliferation.

Endogenous opioids were shown to play an important role in neuronal differentiation via cell migration and growth of neuronal processes, as evidenced by opioid receptor blockade increasing cortical thickness while decreasing neuronal packing density. ${ }^{12}$ In comparison, morphine-exposed mouse brains showed reduced neuronal packing density without changes in cortical thickness. ${ }^{12}$ In addition, a reduction in the total number of neurons in layers II to $\mathrm{V}$ of the somatosensory cortex was measured. ${ }^{12}$ These findings suggest that prenatal morphine exposure not only affects neuronal differentiation, but also that morphine causes neurotoxicity in this region of the brain by either decreasing cell proliferation or inducing cellular death. ${ }^{12}$ 
These observations were conducted on mouse brains extracted on postnatal day 6 , at which point morphine exposed mice already showed neurobehavioral development deficits. ${ }^{12}$

In a similar manner, another study found that prenatal exposure to heroin also had a negative effect on the development of the somatosensory cortex. ${ }^{13}$ Brains of mice transplacentally exposed to heroin showed reductions in the length and number of branches in dendrites of pyramidal neurons. ${ }^{13}$ Once the offspring were born, the aforementioned morphological changes were associated with a reduced tendency to explore objects in novel locations. ${ }^{13}$ These data suggest that prenatal heroin exposure may hinder the development of short-term spatial memory.

One of the proposed mechanisms through which endogenous opioid signaling influences the embryonic development of the central nervous system is through regulation of apoptosis. In vitro studies of embryonic chicks have shown that exposure to opioid receptor agonists results in increased risk for death via brain tissue apoptosis. ${ }^{14}$ More specifically, one study investigated the association between intrauterine morphine exposure and levels of apoptotic markers $\mathrm{Bax}, \mathrm{Bcl} 2$, and caspase 3 during the embryonic development of the central nervous system. ${ }^{15}$ In this study, morphine sulfate was administered to pregnant rats daily until the embryos were extracted on gestational days 9.5 to $13.5 .{ }^{15}$ Immunohistochemical assays on exposed mice embryos showed an overall increase in Bax immunoreactivity and a notable decrease in Bcl2 immunoreactivity on days 10.5 to $12.5 .{ }^{15}$ Overall, intrauterine morphine exposure resulted in the $\mathrm{Bax}$ to $\mathrm{Bcl} 2$ ratio being higher in the study group compared with the control group. ${ }^{15}$ There was also an increase in the amount of cleaved caspase 3 on days 11.5 to $13.5 .{ }^{15}$ These findings suggest that prenatal morphine exposure increases apoptosis in neuroblasts beyond rates observed in normal central nervous system development. Disruptions in physiologic apoptosis regulation may be involved in the morphological changes associated with the neurobehavioral effects observed in neonates and children born to opioid-addicted mothers.

Many of the neurobehavioral sequelae observed in children with prenatal opioid exposure are related to poor memory function, for example, there is an increased prevalence of learning disabilities. ${ }^{5}$ Indeed, studies have noted the effects of prenatal opioid exposure on memory via morphological alterations in the hippocampus and cholinergic system. ${ }^{5}$ Found in the medial temporal lobe of the brain, the hippocampus is known to play a significant role in learning and memory, including spatial memory. ${ }^{16}$ Research on human memory greatly relies on mouse models, with the mice's performance on tasks such as the radial arm ("8-arm”) maze and the Morris maze as reliable indicators of spatial memory function and hippocampus damage. ${ }^{17}$ 
The cholinergic system, and in particular the M1 receptor-a muscarinic acetylcholine receptor subtype-has been noted to have a significant presence in the hippocampus. ${ }^{18}$ When the M1 receptor is activated by acetylcholine, its associated $\mathrm{G}$ protein stimulates downstream mechanisms such as protein kinase $\mathrm{C}(\mathrm{PKC})$ via secondary messengers including inositol triphosphate. It has been found that the expression of M1 receptor mRNA is significantly increased in animals prenatally exposed to heroin. ${ }^{18}$ To investigate any adaptive alterations in downstream M1 receptor signaling, investigators used inositol triphosphate formation as a measure of G-protein activation. ${ }^{18}$ It showed that mouse offspring with prenatal heroin exposure demonstrated significantly higher G-protein activation when compared with the unexposed control. ${ }^{18}$ Similarly, a measured increase in PKC activity in hippocampal tissue was associated with observed increases in the expression of $\mathrm{Gq}$ and $\mathrm{Gi}$ proteins. ${ }^{18}$ The upregulated expression and downstream activity of the M1 receptor in the hippocampus of mice with prenatal heroin exposure is believed to be associated with the reduction of their spatial memory function. ${ }^{18}$

The hyperactivity noted within the hippocampal cholinergic system of prenatally opioid-exposed mice is not limited to the postsynaptic neuron. Another study found that transplacental heroin administration increased the amount of choline transporters in offspring's hippocampal presynaptic neuron terminals. ${ }^{19}$ Evidence of hippocampal cholinergic hyperactivity at both the presynaptic and postsynaptic levels, while the associated behavior of spatial memory continued to be deficient, motivated further investigation on the functional activity of downstream PKC signaling. ${ }^{19}$ It was found that in mice prenatally exposed to heroin, administration of the cholinergic agonist carbachol had no effect on PKC activity in hippocampal neurons. ${ }^{20}$

These studies converge on the suggestion that exogenous opioids must induce some degree of desensitization. Steingart et $\mathrm{al}^{19}$ initially theorized that a desensitization of PKC was the primary event, with a subsequent futile compensatory global hyperactivation. However, a deeper investigation found this not to be the case. When normally functioning embryonic septal cholinergic cells were transplanted into the hippocampus of the study mice, there was complete reversal of physiologic neurologic function as assessed through the 8-arm maze and Morris maze. ${ }^{19}$ The investigators concluded that the prenatal exogenous opioids caused overstimulation of postsynaptic neurons during embryonic synaptogenesis, and this was the primary event leading to the adaptive responses of presynaptic choline transporter upregulation and postsynaptic PKC desensitization. ${ }^{19}$ Overall, this conclusion provides a clear mechanism for some of the neurobehavioral impairments prevalent in children prenatally exposed to opioids.

When discussing the opioid epidemic and the negative effects of prenatal opioid exposure on the embryo's central nervous system, it should be noted that these effects represent neurobehavioral sequelae that continue into 
childhood and beyond. The potential long-term consequences and costs are substantial. Consequently, it is that much more important that current guidelines on the administration and use of opioids during pregnancy be reassessed.

Despite the gravity of the current situation, there is limited evidence of potential ways in which the negative developmental effects of opioids may be reduced. For instance, the aforementioned possibility of an embryonic septal cholinergic cell transplant. In addition, a recent study found that when rats prenatally exposed to opioids are reared in an "enriched environment"-one with exercise equipment, toys, and ample opportunities for socialization-their behavioral and cognitive deficits were mitigated vs rats with the same exposure but reared in control cages, suggesting that a more enriched environment can help alleviate the detrimental effects of prenatal opioid exposure. ${ }^{21}$ Further investigation into opioid-induced neurobehavioral deficit reversal is needed.

\section{Cardiovascular System}

The newborn's central nervous system is not the only major organ system influenced by opioid use during pregnancy. Much like the brain, the embryonic development of the heart is also greatly influenced by endogenous opioids. Opioid growth factors (OGFs) are a crucial element of proper heart development and have been shown to act on the embryonic heart through $\zeta$ opioid receptors to influence heart formation in a direct yet noncytotoxic manner. ${ }^{22,23}$ The opioid growth factor $\left[\mathrm{Met}^{5}\right]$-enkephalin, encoded by the preproenkephalin gene $(P P E)$, has been further characterized as a tightly regulated and crucial OGF for the development of the mammalian heart. ${ }^{22,23}$ $P P E$ mRNA has been identified early on in the rat heart, starting from embryonic day 14 , with its expression initially concentrated in the precardial plate and the aortic sac, and then expanding to the brachial arteries and beyond until birth. ${ }^{22,23}$ As compared with PPE mRNA levels in the adult rat heart, mRNA levels in the fetal rat from embryonic day 16 to postnatal day 1 are anywhere from $33 \%$ to $50 \%$ of the expression found in adults, while $\left[\mathrm{Met}^{5}\right]$ enkephalin is at its highest levels during the later stages of embryonic development and the first 2 weeks of postnatal life. ${ }^{23}$

Acute exposure of rats to [Met $\left.{ }^{5}\right]$-enkephalin during pregnancy results in reduced DNA synthesis in the endoderm, mesoderm, and ectoderm through binding of the OGF to $\zeta$ opioid receptors. ${ }^{24}\left[\mathrm{Met}^{5}\right]$-enkephalin has been shown to decrease DNA synthesis by $30 \%$, while the presence of an antagonist to the $\zeta$ receptor prevented this decrease in DNA synthesis. ${ }^{24}$ Thus, the differential expression of $\left[\mathrm{Met}^{5}\right]$-enkephalin at the various stages of development illustrates its vital role in the DNA synthesis of mononucleated cardiac cells at the embryonic stages of life. ${ }^{22,23}$ These findings indicate that the lower levels of $\left[\mathrm{Met}^{5}\right]$-enkephalin observed in early embryonic stages are 
important for allowing the heart to grow during that time. Similarly, the rise of $\left[\mathrm{Met}^{5}\right]$-enkephalin in later stages of embryonic development suggests an intricate regulation of cardiac cell proliferation as the mammal develops.

The inhibitory role of endogenous opioids on the developing heart has been further demonstrated by the function of $x$ opioid receptors in the neonatal rat. On activation, $x$ opioid receptors inhibit the growth of the ventricular myocyte by decreasing protein content, cell size, and $\left[{ }^{3} \mathrm{H}\right]$ leucine uptake, a marker of new protein synthesis. ${ }^{25}$ Administration of a selective $x$ opioid receptor antagonist has been shown to prevent the decrease in protein content, cell size, and $\left[{ }^{3} \mathrm{H}\right]$ leucine uptake that had originally occurred from the stimulation of $\kappa$ opioid receptors in the developing heart. ${ }^{25}$ In addition to directly acting on the cells of the ventricle and inhibiting growth, $x$ opioid receptors indirectly prevent the development of the ventricular myocyte by blocking norepinephrine-mediated sympathetic stimulation that induces cardiac growth through $\alpha$-and $\beta$-adrenoreceptors. ${ }^{25}$ The way in which $\kappa$ opioid receptors decrease growth of ventricular myocytes, both directly and indirectly, in the neonatal rat illustrates the significant influence of opioids in cardiac development through nuanced control and regulation of growth.

Given the important role opioids play in normal cardiovascular system development, maternal use of opioids may have severe impacts on the embryonic development of a baby's heart. Opioids have been shown to cross the placenta in humans and collect in many different tissues of the fetus, with the heart serving as a major organ for fetal opioid buildup. ${ }^{26}$ In mothers with opioid addiction during pregnancy, fetal heart rates were found to be significantly lower by more than 3 beats per minute (bpm) when compared with the fetal heart rates of nonexposed mothers. From gestational ages 11 weeks to 13 weeks and 6 days, fetal heart rates averaged $156 \mathrm{bpm}$ in opioidexposed fetuses compared with an average of $159.6 \mathrm{bpm}$ in fetuses with nonopioid-dependent mothers. ${ }^{27}$

The lower heart rate of opioid-exposed fetuses indicates that the influence exogenous opioids exert on the development of the embryonic heart and its progression into more advanced stages of functionality is both measurable and significant. These findings may be explained with the aforementioned observations that OGF $\left[\mathrm{Met}^{5}\right]$-enkephalin decreases cardiac DNA synthesis. $^{22,23}$ Such a decrease in cardiac DNA synthesis can prevent the proper proliferation and division of cells in the embryonic heart. Thus, a lack of functional cardiac cells is a possible explanation for the reduced heart rate seen in fetuses with opioid exposure and highlights the way in which the inhibition of cardiac development by opioids leads to the reduced functionality of the fetal heart as a whole. Therefore, there is evidence to support the notion that exogenous opioids can disrupt the tightly regulated process of cardiac proliferation and development, making their use during pregnancy a particularly dangerous risk factor for the fetus. 
Opiate use in parents has been shown to increase the risk for congenital heart defects, providing further evidence that prenatal opioid exposure has significant effects on embryonic heart development. In a study analyzing 225 children with congenital heart defects, $23.5 \%$ had parents addicted to opioids, while only $2.3 \%$ of children with healthy hearts had parents with an opioid addiction. ${ }^{28}$ In addition to opioid addiction, maternal use of opioids as analgesics to alleviate pain has also been linked to congenital heart defects. Analgesic opioid use in early pregnancy, ranging from 1 month before to 3 months after conception, was found to be significantly associated with increased incidence of pulmonary valve stenosis, hypoplastic left heart syndrome, atrial septal defect, conoventricular septal defect, atrioventricular septal defect, and tetralogy of Fallot. ${ }^{29}$ The association between prenatal exposure to opioids and increased incidence of congenital heart defects can be attributed to a lower level of cellular division and differentiation during embryonic cardiovascular development. Again, these findings may be explained by abnormally high opioid concentrations in embryonic tissue decreasing DNA synthesis through increased $\left[\mathrm{Met}^{5}\right]$-enkephalin levels. ${ }^{22,23}$

The direct correlation between opioid use and the incidence of congenital heart defects provides additional support for the notion that opioid use during pregnancy has detrimental, longlasting effects on the fetus. The risk for highly fatal postnatal complications associated with maternal opioid use during pregnancy makes a strong case for the reduction or complete avoidance of opioid intake during pregnancy, while also highlighting the grave risks posed to the fetus if opioid prescriptions are misused.

\section{Conclusions}

With the significant escalation in opioid use in the United States within the last 2 decades as well as the increase of pregnant women using opioids, understanding the consequences of prenatal opioid exposure on fetal development is becoming increasingly important. Opioid use during pregnancy can disrupt numerous physiologic processes involved in reproduction and embryonic development. Starting before fertilization, female opioid users have an observed decrease in fertility rate compared with nonusers. ${ }^{4}$ Specifically, opioids have been shown to prevent the preimplantation embryo from progressing to the blastocyst stage, while also decreasing uterine receptivity to implantation. 6,10 These observations demonstrate the detrimental influence of opioid misuse and abuse from the very beginning of embryonic life.

The development of the fetal brain is impacted by maternal opioid use. While endogenous opioids are important for the proper development of the brain, administration of exogenous opioids can have significant negative consequences. Opioid exposure has been shown to not only hinder the development of somatosensory cortex in mice by causing reduced dendrite length and branching in pyramidal neurons, but also to be neurotoxic by 
decreasing neuronal packing density. ${ }^{12,13}$ Likewise, opioids can have a detrimental impact on the development of the hippocampus and the cholinergic system, leading to impaired neurobehavioral function in neonates with prenatal opioid exposure that may extend into childhood and beyond as poor memory function and learning disabilities. ${ }^{5}$

Use of exogenous opioids during pregnancy also has the potential to exert significant negative effects on the embryonic heart by slowing down the growth of cardiac tissue and interfering in the tightly regulated stages of its development. The decrease in DNA synthesis and cardiac cell proliferation observed with endogenous OGF becomes exaggerated with maternal opioid use, causing a decrease in fetal heart rate and a rise in the incidence of congenital heart defects. ${ }^{22,23,27,28}$ These findings indicate that opioid use during pregnancy can pose life-threatening dangers to the fetus or the newborn.

Determining the association between opioidinduced alterations in embryonic development and the aforementioned neurocognitive or congenital defects is made difficult by numerous confounding variables. Opioid abuse is associated with poor nutrition and sanitary conditions, as well as numerous social factors affecting health and safety. ${ }^{5}$ This is a pertinent consideration as to why most of the studies reviewed in this article used animal models. Animal models allow for stricter control of the confounding variables that could have otherwise posed limitations to the conclusions presented here. Furthermore, animal models prevent the ethical dilemmas of human experimentation, which are especially relevant given the difficulty of making a definitive assessment of overall opioid teratogenicity in humans due to those same confounding variables. The studies reviewed in this article were assessed for internal and external validity, and were not found to pose significant limitations to our conclusions.

Through the study of biochemical alterations caused by prenatal exposure to opioids that may underlie the associated postnatal outcomes, we conclude that opioid use during pregnancy exerts a significantly negative influence on the developing embryo. There is sufficient evidence that opioid use during pregnancy alters fetal development in measurable ways that constitute plausible explanations for the associated life-threatening complications during the postpartum period, as well as serious long-term neurobehavioral consequences. As such, the increasing rates of opioid prescriptions for pregnant women pose serious public health concerns, and the scientific community has an important role in mitigating the consequences of the opioid crisis. Based on this research, it is recommend that the responsible institutions review and reassess guidelines for pharmacological interventions, involving opioids in the pregnant population to ensure that the harmful effects of opioid exposure during fetal development are either eliminated or minimized as much as possible. 


\section{REFERENCES}

1. Guy GP Jr, Zhang K, Bohm MK, et al. Vital Signs: Changes in Opioid Prescribing in the United States, 2006-2015. MMWR Morb Mortal Wkly Rep. 2017;66(26):697-704. doi:10.15585/ mmwr.mm6626a4

2. Desai RJ, Hernandez-Diaz S, Bateman BT, Huybrechts KF. Increase in prescription opioid use during pregnancy among Medicaid-enrolled women. Obstet Gynecol. 2014;123(5):997-1002. doi:10.1097/aog.0000000000000208

3. Muhuri PK, Gfroerer JC, Davies MC. Associations of Nonmedical Pain Reliever Use and Initiation of Heroin Use in the United States. Center for Behavioral Health Statistics and Quality; 2013.

4. Claman AD, Strang RI. Obstetric and gynecologic aspects of heroin addiction. Am J Obstet Gynecol. 1962;83:252-257. https://doi.org/10.1016/0002-9378(62)90639-7.

5. Yanai J, Avraham Y, Levy S, et al. Alterations in septohippocampal cholinergic innervations and related behaviors after early exposure to heroin and phencyclidine. Brain Res Dev Brain Res. 1992;69(2):207214. https://doi.org/10.1016/0165-3806(92)90161-O.

6. Chen Y, Kong S, Tang X, et al. Preimplantation mouse embryo is a target for opioid ligandreceptor signaling. Biol Reprod.2014;91(1):4. doi:10.1095/biolreprod.114.118083

7. Tang X, Chen Y, Ran H, et al. Systemic morphine treatment derails normal uterine receptivity, leading to embryo implantation failure in mice. Biol Reprod. 2015;92(5):118. doi:10.1095/ biolreprod.115.128686

8. Kalyuzhny AE, Hensleigh HC, Arvidsson U, Elde R. Immunocytochemical localization of $\mu$ opioid receptors in follicular cells and preimplantation mouse embryos. Anat Embryol (Berl).

1997;195(5):451-455. doi:10.1007/s004290050064

9. Zhou A, Webb G, Zhu X, Steiner DF. Proteolytic processing in the secretory pathway.J Biol Chem. 1999;274(30):20745-20748. doi:10.1074/jbc.274.30.20745

10. Kawagoe J, Li Q, Mussi P, et al. Nuclear receptor coactivator6 attenuates uterine estrogen sensitivity to permit embryo implantation. Dev Cell. 2012;23(4):858-865. doi:10.1016/ j.devcel.2012.09.002

11. Aloisi AM, Aurilio C, Bachiocco V, et al. Endocrine consequences of opioid therapy. Psychoneuroendocrinology. 2009;34(suppl 1):S162-S168. doi:10.1016/j.psyneuen.2009.05.013

12. Seatriz JV, Hammer RP Jr. Effects of opiates on neuronal development in the rat cerebral cortex. Brain Res Bull. 1993;30(5-6):523-527. doi:10.1016/0361-9230(93)90078-p

13. Lu R, Liu X, Long H, Ma L. Effects of prenatal cocaine and heroin exposure on neuronal dendrite morphogenesis and spatial recognition memory in mice. Neurosci Lett.

2012;522(2):128-133. doi:10.1016/j.neulet.2012.06.023

14. Boronat MA, García-Fuster MJ, García-Sevilla JA. Chronic morphine induces up-regulation of the pro-apoptotic Fas receptor and down-regulation of the anti-apoptotic Bcl-2 oncoprotein in rat brain. BrJ Pharmacol. 2001;134(6):1263-1270. doi:10.1038/sj.bjp.0704364

15. NasiraeiMoghadam S, Kazeminezhad B, Dargahi L, Ahmadiani A. Maternal oral consumption of morphine increases $\mathrm{Bax} / \mathrm{Bcl} 2$ ratio and caspase 3 activity during early neural system development in rat embryos.J Mol Neurosci. 2010;41(1):156164. doi:10.1007/s12031-009-9312-6

16. Burgess N, Maguire EA, O'Keefe J. The human hippocampus and spatial and episodic memory. Neuron. 2002;35(4):625-641. doi:10.1016/s0896-6273(02)00830-9 
17. Vorhees CV, Williams MT. Assessing spatial learning and memory in rodents. ILARJ. 2014;55(2):310-332. doi:10.1093/ilar/ilu013

18. Yanai J, Steingrat RA, Snapir N, Gvaryahu G, Rozenboim I, Katz A. The relationship between neural alterations and behavioral deficits after prenatal exposure to heroin. Ann N Y Acad Sci. 2000;914:402411. https://doi.org/10.1111/j.1749-6632.2000.tb05214.x.

19. Steingart RA, Barg J, Maslaton J, Nesher M, Yanai J. Pre- and postsynaptic alterations in the septohippocampal cholinergic innervations after prenatal exposure to drugs. Brain Res Bull. 1998;46(3):203-209. https://doi.org/10.1016/S0361-9230(97)00454-1.

20. Steingart RA, Abu-Roumi M, Newman ME, Silverman WF, Slotkin TA, Yanai J.

Neurobehavioral damage to cholinergic systems caused by prenatal exposure to heroin or phenobarbital: Cellular mechanisms and the reversal of deficits by neural grafts. Brain Res Dev Brain Res. 2000;122(2):125-133. https://doi.org/10.1016/S0165-3806(00)00063-8.

21. Ahmadalipour A, Sadeghzadeh J, Vafaei AA, Bandegi AR, Mohammadkhani R, Rashidy-Pour A. Effects of environmental enrichment on behavioral deficits and alterations in hippocampal BDNF induced by prenatal exposure to morphine in juvenile rats. Neuroscience. 2015;305:372-383. doi:10.1016/j.neuroscience.2015.08.015

22. McLaughlin PJ, Wu Y. Opioid gene expression in the developing and adult rat heart. Dev Dyn. 1998;211(2):153-163. https://doi.org/10.1002/(SICI)1097-0177(199802)211:2\%3C153::AIDAJA4\%3E3.0.CO;2-G.

23. McLaughlin PJ, Allar MA. Preproenkephalin gene expression and [Met 5]-enkephalin levels in the developing rat heart. Brain Res Mol Brain Res. 1998;60(2):160-167. https://doi.org/10.1016/ S0169-328X(98)00178-8.

24. Zagon IS, Wu Y, McLaughlin PJ. Opioid growth factor and organ development in rat and human embryos. Brain Res. 1999;839(2):313-322. https://doi.org/10.1016/ S0006-8993(99)01753-9.

25. Wang H, Wang G, Yang Y, Wong TM. $x$-Opioid receptor stimulation inhibits growth of neonatal rat ventricular myocytes. Eur J Pharmacol. 2004;498(1-3):53-58. doi:10.1016/ j.ejphar.2004.07.082

26. Ostrea EM Jr, Lynn SM, Wayne RN, Stryker JC. Tissue distribution of morphine in the newborns of addicted monkeys and humans: clinical implications. Dev Pharmacol Ther. 1980;1(2-3):163-170. doi:10.1159/000455532

27. Schmid M, Kuessel L, Klein K, Metz V, Fischer G, KramplBettelheim E. First-trimester fetal heart rate in mothers with opioid addiction. Addiction. 2010;105(7):1265-1268. doi:10.1111/ j.1360-0443.2010.02982.x

28. Sahramian I, Noori NM, Moradi A, Forghani F, Sharafi E. The rate of addiction in parents of toddlers with congenital heart disease. Int J High Risk Behav Addict. 2013;1(4):154-158. doi:10.5812/ijhrba.7312

29. Broussard CS, Rasmussen SA, Reefhuis J, et al. Maternal treatment with opioid analgesics and risk for birth defects. Am J Obstet Gynecol. 2011;204(4):314.e1-314.e11. doi:10.1016/ j.ajog.2010.12.039 